Gut and Liver, Vol. 13, No. 1, January 2019, pp. 77-82

\title{
Plasma Levels of K18 Fragments Do Not Correlate with Alcoholic Liver Fibrosis
}

\author{
Viola Schlossberger ${ }^{1}$, Mathias Worni ${ }^{2}$, Christina Kinm ${ }^{3}$, Matteo Montani ${ }^{4}$, Christian Datz ${ }^{5}$, Jochen Hampe ${ }^{6}$, and Felix \\ Stickel ${ }^{1,7}$ \\ ${ }^{1}$ Hepatology Unit, Clinic Beau-Site Hirslanden, ${ }^{2}$ Department of Visceral Surgery and Medicine, Inselspital, University Clinic of Bern, Bern, \\ ${ }^{3}$ Department of Gastroenterology, Spital Interlaken, Unterseen, ${ }^{4}$ Department of Pathology, University of Bern, Bern, Switzerland, ${ }^{5}$ Department \\ of Internal Medicine, Hospital Oberndorf, Teaching Hospital of the Paracelsus Private University of Salzburg, Oberndorf, Austria, ${ }^{6}$ Medical \\ Department 1, University Hospital Dresden, Technische Universität Dresden, Dresden, Germany, and 'Department of Gastroenterology and \\ Hepatology, University Hospital of Zürich, Zurich, Switzerland
}

Background/Aims: Noninvasive markers of liver fibrosis in alcoholic liver disease (ALD) are crucial to establish early intervention. Previous studies have suggested that plasma levels of cleaved keratin-18 (K18; M30) fragments can predict the severity of liver disease. The aim of this study was to correlate plasma M30 levels with stages of liver fibrosis in ALD. Methods: Patients with ALD ( $n=139,79.1 \%$ males) and liver histology were included, and plasma samples were collected to quantify plasma M30 levels. Patients were stratified into five groups by fibrosis stage ( $F 0=14 ; F 1=15 ; F 2=35 ; F 3=17$; and $F 4=58$ ) according to the Kleiner score. Differences between groups were evaluated using the chi-square test or analysis of variance. Trends by fibrosis stage were calculated by logistic regression analysis, and sensitivity, specificity and positive and negative predictive values were determined. Results: There were no significant differences in M30 levels among fibrosis stages. The correlation between plasma M30 levels and fibrosis was poor (Pearson's correlation coefficient $=0.13$, Spearman $r h o=0.20[p=0.02]$ ), and M30 levels did not correlate with alcohol-specific histological features. However, significant correlations of M30 levels with aspartate aminotransferase (Spearman rho $=0.653, p<0.001$ ) and alanine aminotransferase (Spearman $r h o=0.432, p<0.001$ ) were found. M30 levels of $>200 \mathrm{U} / \mathrm{L}$ reveal a sensitivity for predicting cirrhosis of $84.5 \%$ with a negative predictive value of $73.5 \%$. Conclusions: Plasma M30 levels are often elevated in ALD and correlate with serum transaminases but do not reflect fibrosis. The usefulness as a prognostic marker awaits evaluation in prospective studies. (Gut Liver 2019;13:77-82)
Key Words: Apoptosis; Caspases; Fibrosis progression; Noninvasive diagnosis

\section{INTRODUCTION}

Alcoholic liver disease (ALD) due to excessive alcohol consumption remains one of the major causes of chronic liver disease, ranging from simple steatosis to alcoholic hepatitis, fibrosis/cirrhosis, and hepatocellular carcinoma (HCC). ${ }^{1}$ However, the pathophysiological causes of disease progression still remain poorly understood. Approximately $20 \%$ of patients with alcoholic steatosis progress to fibrosis and cirrhosis, particularly if they continue to drink. ${ }^{2-4}$ Timely diagnosis of the severity of liver damage is crucial to allow for effective counselling and therapeutic interventions. Liver biopsy with histologic evaluation of tissue damage is the gold standard in the differential diagnosis of liver diseases, but they cannot be performed as a follow-up test in short-term intervals, carry a relevant procedure-related risk, and can lead to misclassification due to sampling error. ${ }^{5,6}$ Thus, noninvasive assessment of the severity of alcoholic fibrosis using surrogate markers that reliably reflect the extent of liver scarring, for example, fibrosis, and offer prognostic information is pivotal to allow for risk-guided intervention, easy follow-up during therapy, and risk reduction for patients.

To this end, several noninvasive methods including serum hyaluronic acid levels and several composite scores such as the Enhanced Liver Fibrosis (ELF) score, fibrosis-4 (FIB4) score and aspartate aminotransferase (AST) to platelet ratio index (APRI) were evaluated to assess fibrosis in alcoholic liver damage. However, the positive predictive values for mild and moderate

Correspondence to: Felix Stickel

Department of Gastroenterology and Hepatology, University Hospital of Zürich, Rämistrasse 100, Zürich CH-8091, Switzerland

Tel: +41-31-338 7817, Fax: +41-31-335 3519, E-mail: Felix.stickel@uzh.ch

Received on January 18, 2018. Revised on April 7, 2018. Accepted on April 9, 2018. Published online September 21, 2018

pISSN 1976-2283 eISSN 2005-1212 https://doi.org/10.5009/gnl18037

Viola Schlossberger and Mathias Worni contributed equally to this work as first authors.

@. This is an Open Access article distributed under the terms of the Creative Commons Attribution Non-Commercial License (http://creativecommons.org/licenses/by-nc/4.0) which permits unrestricted non-commercial use, distribution, and reproduction in any medium, provided the original work is properly cited. 
fibrosis remain suboptimal. ${ }^{7}$ More recently, transient elastography (FibroScan) that measures liver stiffness has proven more useful and has become an integral part of the diagnostic armory in the assessment of the severity of fibrosis in ALD.,9

Additionally, earlier studies in patients with nonalcoholic fatty liver disease (NAFLD) indicate that plasma levels of cleaved keratin-18 (K18) fragments predict histological nonalcoholic steatohepatitis (NASH) and severity of disease including fibrosis. ${ }^{10,11}$ K18 is a cytoskeletal protein which represents one of the main intermediate filament peptides in hepatocytes. During hepatocellular cell death, K18 is released from decaying hepatocytes into the blood stream. During apoptotic cell death, K18 is cleaved by caspases and the resulting cleavage products can be captured using an enzyme-linked immunosorbent assay (ELISA). While levels of cleaved K18 fragments (referred to as M30 levels in this manuscript) are considered relatively specific for apoptotic cell death, non-cleaved K18 levels (M65) reflect total cell death from both apoptosis and necrosis. ${ }^{12}$ Subsequent studies showed that although plasma M30 levels have a high specificity for NAFLD and associated fibrosis, its usefulness as a screening test for staging NASH was limited due to low sensitivity, ${ }^{13}$ and a later meta-analysis confirmed a sensitivity of only $66 \%$ and a moderately good specificity of only $82 \%$ of M30 plasma levels in diagnosing NASH. ${ }^{14}$

Regarding ALD, the diagnostic value of levels of M30 levels has been studied only in one very recent study. Mueller and co-workers measured serum concentrations of total K18 fragments (M65) and caspase-cleaved M30 levels in actively drinking alcoholic patients referred for alcohol detoxification who also underwent liver biopsy. ${ }^{15}$ Authors considered a panel of key laboratory and histological features of ALD and found significant correlations between both, M65 and M30 levels, with histological apoptosis, ballooning, Mallory-Denk bodies, and steatosis, but interestingly, less so with fibrosis. However, the latter is crucial since fibrosis at any stage is considered the hallmark lesion in predicting progression to cirrhosis. ${ }^{16,17}$ To validate the diagnostic usefulness of plasma M30 levels to noninvasively assess alcoholic liver fibrosis, we assessed their correlation with fibrosis stages in a cohort of patients with ALD and available liver histology.

\section{MATERIALS AND METHODS}

\section{Patients and samples}

The study protocol was approved by the Cantonal Ethic Committee of Bern (No. KEK BE 062/11), Switzerland, and all patients gave written informed consent prior to inclusion for the present study. Alcoholic patients were included into the study (Inselspital, $\mathrm{n}=74$; Clinic Beau-Site, $\mathrm{n}=38$ ) once they became abstinent, and if they met the following criteria: (1) liver disease due to excessive alcohol consumption; (2) exclusion of other causes of liver disease; (3) available serum samples with match- ing standard liver enzyme values; and (4) available liver biopsy.

All subjects were assessed for their previous individual drinking behavior. Liver damage was considered as alcohol-related in those with a reported alcohol history of $>60 \mathrm{~g} /$ daily in males and $>30 \mathrm{~g} /$ daily in females. Patients who were positive for hepatitis B surface antigen, anti-hepatitis C IgG, anti-nuclear antibodies above 1:80 and anti-mitochondrial antibodies above $1: 40$, and reported regular (daily) cannabis consumption were excluded. All patients who presented with elevated serum ferritin levels together with an increased transferrin saturation (>50\%) were genotyped for hemochromatosis gene mutations, and excluded if found homozygous for the C282Y mutation. To exclude patients with any severe extrahepatic inflammatory or infectious condition, serum levels of C-reactive protein had to be below $20 \mathrm{mg} / \mathrm{L}$ at the time of inclusion into the study. All patients underwent a percutaneous liver biopsy, and histology was examined according to Kleiner et al. ${ }^{18}$ This score reflects the extent of histological damage of hepatocytes, combining the degree of fibrosis on a 5-point scale from 0 to 4 , and the degree of inflammatory activity on a 4-point scale from A0 to A3. Patients were then divided into five subgroups according to their Kleiner-score according to the fibrotic stage (F0, F1, F2, F3, and F4). Fibrosis was visualized by Sirius Red staining applying standard protocols. Further, plasma M30 levels were measured in 29 healthy control subjects (75.9\% male) with no evidence of liver injury and only occasional alcohol consumption.

Plasma samples were collected from a peripheral blood draw at the Department of Visceral Surgery and Medicine, Inselspital Bern and the Hepatology Unit, Klinik Hirslanden BeauSite, Bern, both in Switzerland, and stored at $-80^{\circ} \mathrm{C}$ until being processed. Quantification of plasma M30 levels was performed using the M30-Apoptosense ELISA according to the manufacturer's instructions (Catalog Prod No.10010; Peviva AB, Axxora $\mathrm{GmbH}$, Germany). Results are provided in serum M30 levels as units per liter with an upper limit of normal (ULN) of $200 \mathrm{U} / \mathrm{L} .^{14}$ All values were determined in duplicates.

All other laboratory variables were determined by means of routine diagnostic procedures established in the two hospitals. Reference ranges for routine laboratory values were identical in the two hospitals.

\section{Statistics}

Statistical analyses were performed using STATA 15.1. Data are expressed as means ( \pm standard deviation [SD]) or count $(\%)$ as indicated. The statistical significance of differences was evaluated using chi-square test or the nonparametric analysis of variance Kruskal-Wallis test. To assess trends over fibrosis stage, logistic regression was performed using fibrosis stage as continuous variable as predictor. To assess correlations between variables, the Pearson's correlation coefficient and the Spearman rho were calculated. Sensitivity, specificity, positive and negative predictive values were calculated for M30 levels and 
liver cirrhosis (F4) and severe fibrosis (F2-4). A two-sided pvalue of $<0.05$ was considered statistically significant.

\section{RESULTS}

In the present study, 139 consecutive patients with ALD referred to our liver clinic for diagnostic work-up were included (Inselspital, $n=74$; Clinic Beau-Site, $n=38$ ). Mean age of the patients was 57.6 years (SD, 10.1) while 110 patients were male (79.1\%) (Table 1). Most patients had a fibrosis stage of F4 (51.7\%) followed by F2 (25.2\%). While the groups were relatively wellmatched for gender, age and body mass index, patients with fibrosis stage F2 and 3 had higher AST levels (mean, 101.2 U/L; $\mathrm{SD}, 82.1$ ) than those with no or minimal fibrosis (mean, 60.1 U/L; $\mathrm{SD}, 49.6)(\mathrm{p}=0.02)$. There was no difference for ALT levels (mean, 112.5 U/L; SD, 23.5; mean, 62.0 U/L; SD, 70.9; respectively, $\mathrm{p}=0.13$ ) between those two groups. Mean plasma M30 levels in healthy control subjects were $164 \mathrm{U} / \mathrm{L}$ (SD, 46.9). There was a trend towards higher plasma M30 levels with increasing fibrosis stages, but there was a large variation within groups ( $p$ for trend=0.14) (Table 1, Fig. 1). According to this variability, our data demonstrate only a small strength of correlation between M30 levels and fibrosis (Pearson's correlation coefficient, 0.13; Spearman $r h o=0.20[p=0.02]$ ) (Fig. 1). Besides the large variation of M30 levels within individual fibrosis stage groups, there was also no significant difference between the fibrosis stages $(\mathrm{p}=0.44)$ (Fig. 1). There was a trend that mean M30 levels were higher among patients with significant fibrosis $(\geq F 2)$ (mean, 674 $\mathrm{U} / \mathrm{L} ; \mathrm{SD}, 679$ ) compared to patients without significant fibrosis (F0 and F1) (mean, $411 \mathrm{U} / \mathrm{L} ; \mathrm{SD}, 552$ ), but this failed to reach significance $(\mathrm{p}=0.06)$.

However, we found a significant correlation of serum M30 levels with AST (Spearman rho=0.653, $\mathrm{p}<0.001$ ) and with ALT (Spearman rho $=0.432, \mathrm{p}<0.001$ ) (Fig. 2). Considering an ULN for serum M30 levels of $200 \mathrm{U} / \mathrm{L}$, the proportions of patients with normal M30 levels within the different fibrosis stage groups F0 to 4 were $43 \%, 33 \%, 31 \%, 18 \%$, and $16 \%$, respectively ( $\mathrm{p}$ for trend=0.01). The mean AST level among patients with normal M30 levels was 42.1 U/L (SD, 32.7) while it was 116.2 U/L (SD, 220.8) among patients with elevated M30 levels ( $p=0.05$ ). Mean ALT levels were higher among patients with elevated M30 levels (87.7 U/L; SD, 134.4) compared to those with normal M30 levels (37.1 U/L; SD, 37.6; $\mathrm{p}=0.03$ ).

There was no correlation between M30 levels and other key lesions of alcoholic liver injury including ballooning, steatosis, inflammatory activity or the presence of Mallory-Denk bodies (data not shown).

Sensitivity of the cutoff value of 200 U/L to predict liver cir-

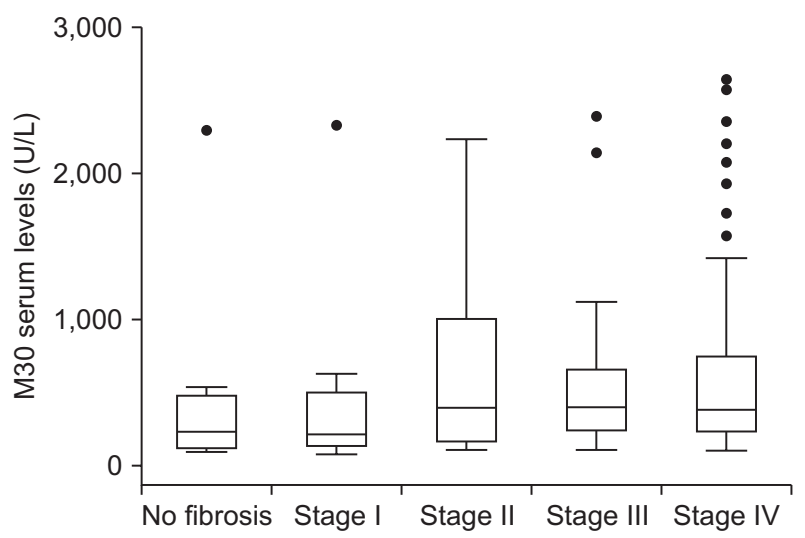

Fig. 1. M30 serum levels in patients with alcoholic liver disease as assessed histologically for fibrosis by Kleiner et al. ${ }^{18}$ (no fibrosis=14; fibrosis stage $\mathrm{I}=15$; stage $\mathrm{II}=35$; stage $\mathrm{III}=17$; and stage $\mathrm{IV}=58$ ).

Table 1. Demographics and Plasma M30 Levels

\begin{tabular}{|c|c|c|c|c|c|c|}
\hline \multicolumn{6}{|c|}{ Fibrosis stage } & \multirow{2}{*}{ p-value ${ }^{*}$} \\
\hline & F0 & $\mathrm{F} 1$ & F2 & F3 & F4 & \\
\hline No. of patients & $14(10.1)$ & $15(10.8)$ & $35(25.2)$ & $17(12.2)$ & $58(51.7)$ & \\
\hline Age, yr & $59.1 \pm 8.1$ & $56.2 \pm 13.5$ & $57.8 \pm 10.1$ & $54.2 \pm 10.7$ & $58.5 \pm 9.4$ & $0.56^{*}$ \\
\hline Sex & & & & & & 0.94 \\
\hline Female & $2(14.3)$ & $4(26.7)$ & $7(20.0)$ & $4(23.5)$ & $12(20.7)$ & \\
\hline Male & $12(85.7)$ & $11(73.3)$ & $28(80.0)$ & $13(76.5)$ & 46 (79.3) & \\
\hline BMI, $\mathrm{m}^{2} / \mathrm{kg}$ & $26.3 \pm 2.6$ & $26.4 \pm 3.2$ & $24.8 \pm 4.7$ & $24.6 \pm 1.9$ & $27.4 \pm 5.8$ & 0.12 \\
\hline ALT, U/L & $47 \pm 34.7$ & $76.1 \pm 92.2$ & $111.6 \pm 166.5$ & $114.4 \pm 181.0$ & $48.7 \pm 68.1$ & 0.07 (p-trend, 0.40) \\
\hline AST, U/L & $56.4 \pm 52.3$ & $63.5 \pm 48.6$ & $97.0 \pm 73.4$ & $109.8 \pm 99.6$ & $114.3 \pm 289.5$ & 0.82 (p-trend, 0.23) \\
\hline M30, U/L & $409.9 \pm 567.9$ & $413.0 \pm 556.5$ & $669.0 \pm 659.2$ & $727.3 \pm 792.8$ & $661.8 \pm 666.3$ & 0.44 (p-trend, 0.14) \\
\hline M30 > 200 U/L & 8 (57.1) & $10(66.7)$ & 24 (68.6) & $14(82.4)$ & $49(84.5)$ & 0.13 (p-trend, 0.01) \\
\hline
\end{tabular}

Data are presented as number $(\%)$ or mean \pm SD.

BMI, body mass index; ALT, alanine aminotransferase; AST, aspartate aminotransferase.

*Chi-square test.

Fibrosis stage as assessed by Kleiner et al. ${ }^{18}$ 


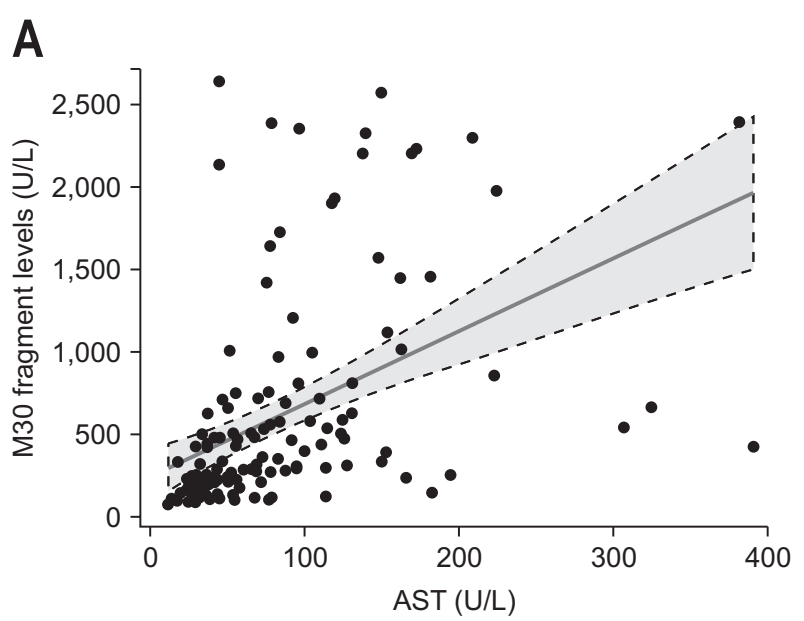

Fig. 2. (A) Correlation of K18 fragments with aspartate aminotransferase (AST). (B) Correlation of K18 fragments with alanine aminotransferase (ALT). Gray-shaded area refers to confidence interval.

rhosis (F4) was $84.5 \%$ while its specificity was only $30.9 \%$. The positive predictive value was $46.7 \%$ with a negative predictive value of 73.5\%. To predict at least severe fibrosis (F2-4), the sensitivity of the cutoff value of $200 \mathrm{U} / \mathrm{L}$ was $79.1 \%$ with a specificity of $37.9 \%$. The positive predictive value was $82.9 \%$ with a negative predictive value of $32.4 \%$.

\section{DISCUSSION}

The presented data clearly demonstrate that M30 levels >200 U/L are often found among patients with ALD with increasing severity of chronic liver damage as reflected by histological fibrosis. M30 levels also correlate with serum AST levels andalbeit to a lesser extent-with hepatocyte-specific ALT activities. However, in a relevant proportion of 15\% to 31\% of patients with ALD, serum M30 levels were low despite the presence of relevant fibrosis (F2-F4) and elevated serum liver enzymes. Our data comply with the data by Mueller et al. ${ }^{15}$ who demonstrated similar correlations of M30 levels with serum liver enzyme levels. In as such, M30 levels can be considered relatively specific for alcohol-mediated liver injury in which AST elevations are often more profound than those of ALT, resulting in an elevation of the so-called De Ritis ratio above $1 .{ }^{19}$ Importantly, plasma M30 levels represent circulating K18 fragments cleaved by caspases from the cell membranes of epithelial cells and appear in peripheral blood when these cells undergo apoptosis. For this reason, common cognition is that elevated M30 levels reflect ongoing apoptosis/cell death rather than inflammation and/or fibrosis. $^{20}$

Unlike in the study by Mueller et $a l,{ }^{15}$ we found no significant correlation between M30 levels and the presence of ballooning, steatosis, inflammatory activity or Mallory-Denk bodies. This discrepancy may be due to the fact that patients in the Mueller et al. study underwent a liver biopsy while still being active drinkers or shortly thereafter, a time point when apoptosis

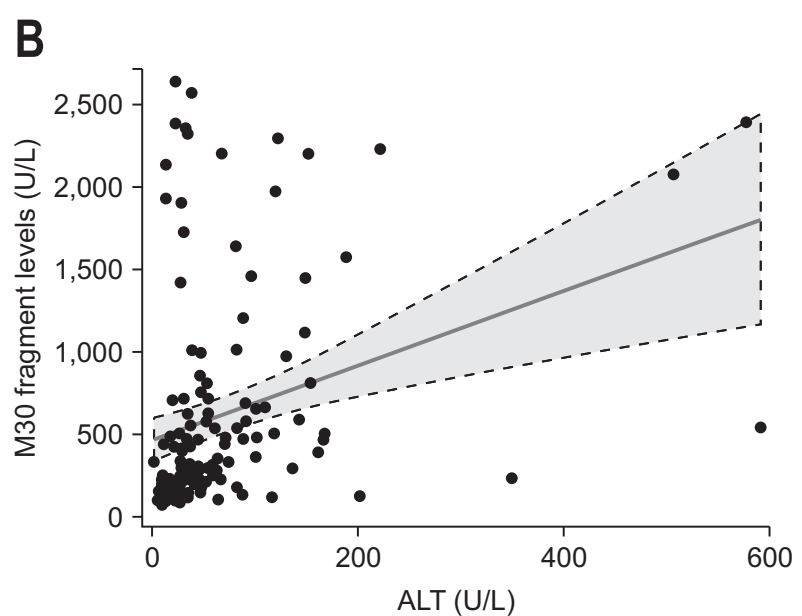

might have been most pronounced or still ongoing. However, our patients were biopsied upon abstinence and much of the preexisting apoptosis may have disappeared by the time of the biopsy. Another reason for the lack of correlation with key features of ALD in our study could be the usage of routine biopsies in which we performed no immunohistochemistry staining for apoptosis or K8/K18, the two single features, which showed the strongest correlation with M30 levels in the study performed by Mueller et al. ${ }^{15}$

So far, the present study is only the second clinical report investigating the diagnostic utility of circulating plasma levels of M30, for example, caspase-cleaved K18 fragments, in various fibrosis stages of ALD. Our essentially negative findings with regard to a possible association with fibrosis, however, provide interesting information in as such they show that plasma M30 levels reflect hepatocellular damage mirrored by elevated serum transaminases. Here, M30 levels correlate slightly better with AST levels than with ALT, and therefore seem to reflect hepatocyte damage just as serum transaminases do. The positive correlation between serum M30 levels and plasma liver enzyme activities has also been demonstrated by other researchers. For example, Sgier et al. ${ }^{21}$ showed the positive impact of a successful antiviral therapy on circulating M30 levels in patients with chronic hepatitis C. Although correlations between M30 levels and serum transaminase levels were significant but weak, therapy responders showed both decreasing liver enzyme and M30 levels.

More recent data from the CANONIC study show that plasma M30 and M65 levels are increased with increasing severity in cirrhotic patients with acute decompensation or acute on chronic liver failure. ${ }^{22}$ Interestingly and confirming our data, a background of alcohol abuse was associated with increased cell death markers whereas underlying infection was not. It has to be emphasized that infection as a potential confounder in our cohort was excluded prior to inclusion of patients into the 
study. McDonald and coworkers demonstrated a close correlation between the cell death markers M30 and M65 and markers of systemic inflammation, hepatic failure, ALT and bilirubin but not with markers of extrahepatic organ injury. The significance of keratins as type II acute-phase responsive genes had been convincingly demonstrated in a clinical study using liver biopsies from patients with ALD, nonalcoholic steatohepatitis, chronic hepatitis B and C and from control subjects by measuring hepatic mRNA expression of K7, K8, K18 and K19. ${ }^{23}$ All keratins were overexpressed 1.5- to 3-fold, particularly in subjects with moderate versus minimal inflammation, and K8 and K18 were closely correlated with ALD.

Interestingly, the same group of researchers also identified polymorphic variants in the genes coding for K18 and K8 as genetic susceptibility loci for acute liver failure ${ }^{24}$ and severely progressing primary biliary cholangitis, ${ }^{25}$ adding to the persuasion that the gene products, $\mathrm{K} 8$ and $\mathrm{K} 18$, play indeed a role in the progression of chronic fibrosing liver disease, and possibly, associated complications. Regarding the latter, Mueller et al. ${ }^{15}$ have suggested that both elevated M30 and M65 levels are predictors of increased non-HCC liver-related mortality with cutoff values of M65 levels >650 U/L and M30 > 255 U/L, respectively. This assumption is supported by another recent study which determined whether M65 or M30 plasma levels were different between patients with alcoholic cirrhosis and alcoholic hepatitis. ${ }^{26}$ Compared to alcoholic cirrhotics, patients with alcoholic hepatitis revealed significantly higher M30 and up to 5-fold higher M65 levels, and the M30/M65 ratio outscored the Model for End-stage Liver Disease and age, serum bilirubin, INR, and serum creatinine (ABIC) prognostic scores to predict survival.

In a similar but larger recent clinical study, Bissonnette et al. ${ }^{27}$ tested the diagnostic utility of M30 and M65 plasma levels to identify patients with severe alcoholic hepatitis. Both M30 and M65 levels were elevated in alcoholic hepatitis patients, and both markers had an area under the receiver operating characteristics curve of 0.84 to estimate the presence of AH. Particularly good was the performance of M65 with positive and negative predictive values of $91 \%$ and $88 \%$, respectively, for the presence of histological alcoholic hepatitis.

The bottom line of these and our data is that M30 levels are often elevated in ALD, and likely in other chronic liver diseases. It shows a significant correlation with serum transaminase levels and thus reflects acute hepatocyte damage, but a close correlation to the fibrotic stage could not be shown so far. However, as an additional application, M30 levels may be used as a prognostic marker to predict mortality, although it remains open how an elevated marker of acute liver injury translates into a prediction of long-term prognosis in ALD. Clearly, more research on the causes of death in those with elevated M65/M30 levels is required to dissect liver-related from non-liver related causes of increased mortality.

It has to be conceded that our study has several weaknesses and limitations. First, we have no data on M65 plasma or serum levels, since this was not considered relevant at the time the study was conceived. Retrospective re-analysis of stored plasma samples is not an option since these samples are no longer available. Second, we have no long-term prospective data on the course of the disease of the included patients which prevents any conclusions on the value of M30 as a predictor of mortality or survival, respectively. Also, liver lesions other than fibrosis were not further explored by additional investigational steps such as specific immunohistology stainings. At the time the study was initiated, expectations towards M30 as a marker of fibrosis prevailed all other possible applications.

However, strengths of our data are that all patients had available liver histology with scored fibrosis stages in all, and matched M30 measurements. Our study cohort has a considerable size in a chronic liver entity that is less frequently studied. We attest that the M30 level at the present time has no role as a fibrosis marker, but rather serves as a marker of acute liver damage similar to serum liver transaminases. Further studies should elucidate the potential role of M30 and M65 as a predictor of mortality in alcoholic cirrhosis and alcoholic hepatitis in prospective studies to establish its usefulness as a prognostic marker, possibly also to improve organ allocation in liver transplantation programs.

\section{CONFLICTS OF INTEREST}

No potential conflict of interest relevant to this article was reported.

\section{ACKNOWLEDGEMENTS}

The present work has been funded in part by funds from the Swiss Foundation for Alcohol Research (SSA grant 261/15 to F.S.) and the Swiss National Funds (SNF 310030_138747 and 310030_169196; to F.S.).

Author contributions: V.S., wrote manuscript, re-analyzed the raw data, and approved the final version of the manuscript; M.W., wrote manuscript, performed the statistical analysis, critically revised the manuscript and approved its final version; C.K., processed the samples, obtained patients' information and the experimental data, and approved the final version of the manuscript; M.M., assessed and scored liver histologies, and approved the final version of the manuscript; J.H., conceived the study protocol, critically revised the manuscript, and approved its final version; C.D., contributed to the drafting and critical revision of the manuscript, and approved its final version; F.S., conceived the study protocol, obtained ethics approval, recruited patients, wrote the manuscript and approved its final version. 


\section{REFERENCES}

1. Stickel F, Datz C, Hampe J, Bataller R. Pathophysiology and management of alcoholic liver disease: update 2016. Gut Liver 2017;11:173-188.

2. Teli MR, Day CP, Burt AD, Bennett MK, James OF. Determinants of progression to cirrhosis or fibrosis in pure alcoholic fatty liver. Lancet 1995;346:987-990.

3. Kamper-Jørgensen M, Grønbaek M, Tolstrup J, Becker U. Alcohol and cirrhosis: dose--response or threshold effect? J Hepatol 2004;41:25-30.

4. Rehm J, Taylor B, Mohapatra S, et al. Alcohol as a risk factor for liver cirrhosis: a systematic review and meta-analysis. Drug Alcohol Rev 2010;29:437-445.

5. Bedossa P, Dargère D, Paradis V. Sampling variability of liver fibrosis in chronic hepatitis C. Hepatology 2003;38:1449-1457.

6. Cadranel JF, Rufat P, Degos F. Practices of liver biopsy in France: results of a prospective nationwide survey. For the Group of Epidemiology of the French Association for the Study of the Liver (AFEF). Hepatology 2000;32:477-481.

7. Chrostek L, Panasiuk A. Liver fibrosis markers in alcoholic liver disease. World J Gastroenterol 2014;20:8018-8023.

8. Nguyen-Khac E, Chatelain D, Tramier B, et al. Assessment of asymptomatic liver fibrosis in alcoholic patients using fibroscan: prospective comparison with seven non-invasive laboratory tests. Aliment Pharmacol Ther 2008;28:1188-1198.

9. Mueller S, Millonig G, Sarovska L, et al. Increased liver stiffness in alcoholic liver disease: differentiating fibrosis from steatohepatitis. World J Gastroenterol 2010;16:966-972.

10. Feldstein AE, Wieckowska A, Lopez AR, Liu YC, Zein NN, McCullough AJ. Cytokeratin-18 fragment levels as noninvasive biomarkers for nonalcoholic steatohepatitis: a multicenter validation study. Hepatology 2009;50:1072-1078.

11. Joka D, Wahl K, Moeller S, et al. Prospective biopsy-controlled evaluation of cell death biomarkers for prediction of liver fibrosis and nonalcoholic steatohepatitis. Hepatology 2012;55:455-464.

12. Kramer G, Erdal H, Mertens HJ, et al. Differentiation between cell death modes using measurements of different soluble forms of extracellular cytokeratin 18. Cancer Res 2004;64:1751-1756.

13. Cusi K, Chang Z, Harrison S, et al. Limited value of plasma cytokeratin-18 as a biomarker for NASH and fibrosis in patients with non-alcoholic fatty liver disease. J Hepatol 2014;60:167-174.

14. Kwok R, Tse YK, Wong GL, et al. Systematic review with meta- analysis: non-invasive assessment of non-alcoholic fatty liver disease: the role of transient elastography and plasma cytokeratin-18 fragments. Aliment Pharmacol Ther 2014;39:254-269.

15. Mueller S, Nahon P, Rausch V, et al. Caspase-cleaved keratin-18 fragments increase during alcohol withdrawal and predict liverrelated death in patients with alcoholic liver disease. Hepatology 2017;66:96-107.

16. Mathurin P, Beuzin F, Louvet A, et al. Fibrosis progression occurs in a subgroup of heavy drinkers with typical histological features. Aliment Pharmacol Ther 2007;25:1047-1054.

17. Bataller R, Gao B. Liver fibrosis in alcoholic liver disease. Semin Liver Dis 2015;35:146-156.

18. Kleiner DE, Brunt EM, Van Natta M, et al. Design and validation of a histological scoring system for nonalcoholic fatty liver disease. Hepatology 2005;41:1313-1321.

19. De Ritis F, Coltorti M, Giusti G. An enzymic test for the diagnosis of viral hepatitis; the transaminase serum activities. Clin Chim Acta 1957;2:70-74

20. Malhi H, Gores GJ. Cellular and molecular mechanisms of liver injury. Gastroenterology 2008;134:1641-1654.

21. Sgier C, Müllhaupt B, Gerlach T, et al. Effect of antiviral therapy on circulating cytokeratin-18 fragments in patients with chronic hepatitis C. J Viral Hepat 2010;17:845-850.

22. Macdonald S, Andreola F, Bachtiger P, et al. Cell death markers in patients with cirrhosis and acute decompensation. Hepatology 2018;67:989-1002.

23. Guldiken N, Usachov V, Levada K, et al. Keratins 8 and 18 are type II acute-phase responsive genes overexpressed in human liver disease. Liver Int 2015;35:1203-1212.

24. Strnad P, Zhou Q, Hanada S, et al. Keratin variants predispose to acute liver failure and adverse outcome: race and ethnic associations. Gastroenterology 2010;139:828-835.

25. Zhong B, Strnad P, Selmi C, et al. Keratin variants are overrepresented in primary biliary cirrhosis and associate with disease severity. Hepatology 2009;50:546-554.

26. Woolbright BL, Bridges BW, Dunn W, Olson JC, Weinman SA, Jaeschke H. Cell death and prognosis of mortality in alcoholic hepatitis patients using plasma keratin-18. Gene Expr 2017;17:301-312.

27. Bissonnette J, Altamirano J, Devue C, et al. A prospective study of the utility of plasma biomarkers to diagnose alcoholic hepatitis. Hepatology 2017;66:555-563. 